\title{
Bulk and Surface Electronic Structure of the Dual-Topology Semimetal $\mathrm{Pt}_{2} \mathrm{HgSe}_{3}$
}

\author{
I. Cucchiø, ${ }^{1}$ A. Marrazzo $\odot,{ }^{2}$ E. Cappelli, ${ }^{1}$ S. Riccò, ${ }^{1}$ F. Y. Bruno $\odot,{ }^{1,3}$ S. Lisi, ${ }^{1}$ M. Hoesch $\odot,{ }^{4,5}$ T. K. Kim $\odot,{ }^{4}$ C. Cacho, ${ }^{4}$ \\ C. Besnard, ${ }^{1}$ E. Giannini $\odot,{ }^{1}$ N. Marzari $\odot,{ }^{2}$ M. Gibertini $\odot,{ }^{1,2}$ F. Baumberger $\odot,{ }^{1,6}$ and A. Tamai $\odot^{1, *}$ \\ ${ }^{1}$ Department of Quantum Matter Physics, University of Geneva, 24 quai Ernest Ansermet, CH-1211 Geneva, Switzerland \\ ${ }^{2}$ Theory and Simulation of Materials (THEOS), and National Centre for Computational Design \\ and Discovery of Novel Materials (MARVEL), École Polytechnique Fédérale de Lausanne, CH-1015 Lausanne, Switzerland \\ ${ }^{3}$ GFMC, Departamento de Física de Materiales, Universidad Complutense de Madrid, 28040 Madrid, Spain \\ ${ }^{4}$ Diamond Light Source, Harwell Campus, Didcot OX11 ODE, United Kingdom \\ ${ }^{5}$ Deutsches Elektronen-Synchrotron DESY, Photon Science, 22607 Hamburg, Germany \\ ${ }^{6}$ Swiss Light Source, Paul Scherrer Institute, CH-5232 Villigen, Switzerland
}

(Received 13 September 2019; accepted 14 February 2020; published 13 March 2020)

\begin{abstract}
We report high-resolution angle-resolved photoemission measurements on single crystals of $\mathrm{Pt}_{2} \mathrm{HgSe}_{3}$ grown by high-pressure synthesis. Our data reveal a gapped Dirac nodal line whose (001) projection separates the surface Brillouin zone in topological and trivial areas. In the nontrivial $k$-space range, we find surface states with multiple saddle points in the dispersion, resulting in two van Hove singularities in the surface density of states. Based on density-functional theory calculations, we identify these surface states as signatures of a topological crystalline state, which coexists with a weak topological phase.
\end{abstract}

DOI: 10.1103/PhysRevLett.124.106402

The prediction of the quantum spin Hall effect in graphene by Kane and Mele triggered a reformulation of band theory incorporating the concept of topology [1,2]. Demonstrated first in $\mathrm{HgTe} / \mathrm{CdTe}$ quantum well structures $[3,4]$, a quantum spin Hall state has subsequently been identified in exfoliated $1 T^{\prime} \mathrm{WTe}_{2}$ [5-8] and was reported in bismuthene grown on $\mathrm{SiC}$ [9]. Recently, a robust quantum spin Hall insulator (QSHI) with a gap of up to $0.5 \mathrm{eV}$ has also been predicted in monolayer (ML) $\mathrm{Pt}_{2} \mathrm{HgSe}_{3}$ [10], raising the possibility of a highly insulating state up to room temperature in a van der Waals material.

When stacked to form a three-dimensional (3D) crystal, a 2D QSHI generically turns into a weak topological insulator (WTI) with no protected states on the top and bottom surface of the crystal [11]. Intriguingly though, recent theoretical work found a far richer scenario for bulk $\mathrm{Pt}_{2} \mathrm{HgSe}_{3}$. References [12,13] predicted that $\mathrm{Pt}_{2} \mathrm{HgSe}_{3}$ is one of only a few known dual-topological insulators and may host different surface states protected by symmetries that are unrelated to the QSHI state [14-17]. Specifically, $\mathrm{Pt}_{2} \mathrm{HgSe}_{3}$ was found to be a topological crystalline insulator (TCI) arising from a threefold mirror symmetry, in addition to a weak topological insulator protected by the preservation of translational symmetry in the stacking of the layers. However, to date little is known from experiment about the bulk band structure supporting the different topological phases of $\mathrm{Pt}_{2} \mathrm{HgSe}_{3}$ [18] and the key signature of the TCI, a topological surface state on the (001) surface, has not been reported.

Here we present an angle-resolved photoemission (ARPES) study of cleaved bulk $\mathrm{Pt}_{2} \mathrm{HgSe}_{3}$ single crystals.
We experimentally observe a spin-split surface state with multiple saddle point singularities on the (001) surface and show how this state emerges from a nodal line gapped by spin-orbit interactions. Based on first principles calculations, we identify this surface state as the signature of a TCI phase coexisting with the generic WTI phase found in the same calculations. Our Letter thus provides evidence for the dual topology of $\mathrm{Pt}_{2} \mathrm{HgSe}_{3}$.

We start by describing the growth of jacutingaite $\mathrm{Pt}_{2} \mathrm{HgSe}_{3}$ single crystals. Since $\mathrm{Hg}$ is highly volatile, we employed a high-pressure synthesis route using a hightemperature cubic-anvil press. Applying pressure in the gigapascal range prevents $\mathrm{Hg}$ from boiling off and allowed us to stabilize a stoichiometric melt at temperatures as high as $900-1000^{\circ} \mathrm{C}$. Elemental Pt (purity $99.98 \%$ ) and Se (purity 99.999\%), and the compound $\mathrm{HgSe}$ (purity 99.9\%), were thoroughly mixed with a $\mathrm{Hg}$-exceeding nominal composition $\mathrm{Pt}_{2} \mathrm{Hg}_{1.1} \mathrm{Se}_{3}$ and pelletized under Ar atmosphere inside a glovebox. The pellet was inserted in a boron nitride crucible surrounded by a graphite heater that tightly filled the pyrophillite pressure cell [Fig. 1(a)]. The cell was first pressurized to $1.5-2 \mathrm{GPa}$, then heated at a fast rate to the target temperature $850-1000{ }^{\circ} \mathrm{C}$, maintained at high temperature for $1-2 \mathrm{~h}$ then slowly cooled down $\left(50-75^{\circ} \mathrm{C} / \mathrm{h}\right)$ to $650^{\circ} \mathrm{C}$ followed by a quench to room temperature. Crystalline platelets forming inside the solidified pellet could then be extracted mechanically [Figs. 1(b) and 1(c)]. The crystal sizes obtained by this procedure were in the range of $0.5-1 \mathrm{~mm}$ in the lateral dimension and $50-100 \mu \mathrm{m}$ in thickness. Crystals were found to have a trigonal unit cell (space group $P \overline{3} m 1$ ) with lattice 
(a)

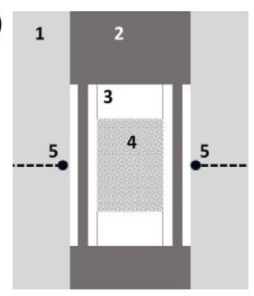

(b)

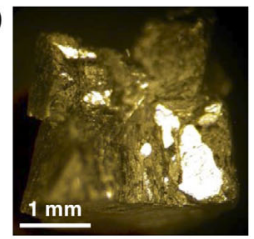

(d)
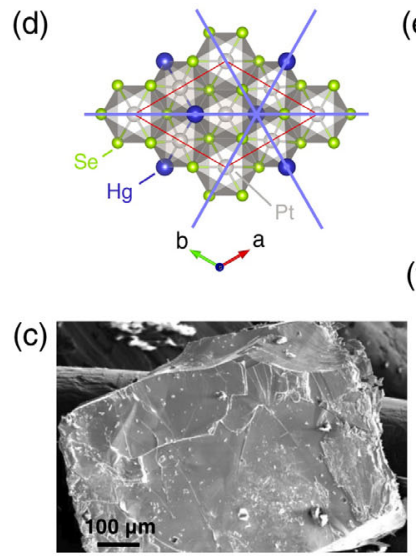

(e)

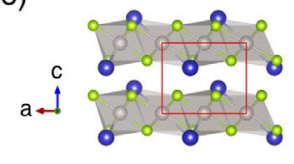

(f)

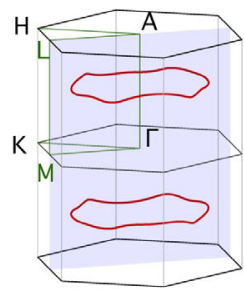

(g)

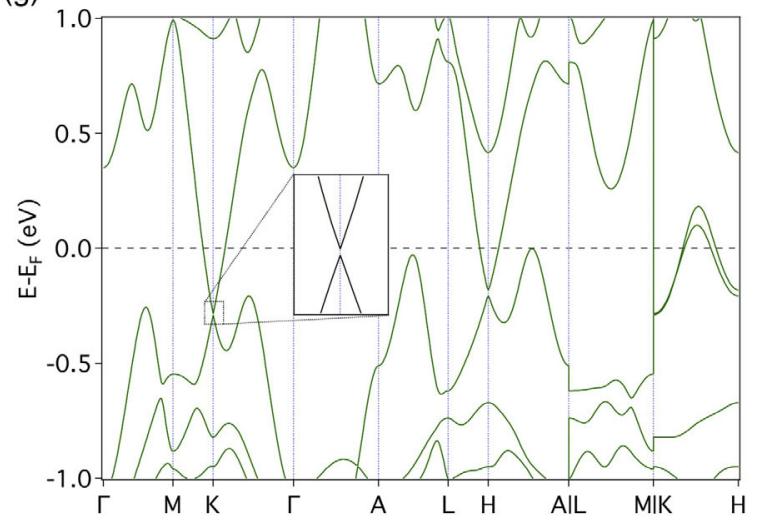

FIG. 1. Bulk jacutingaite single crystals. (a) Sketch of the high-pressure cell: 1, pyrophyllite cell; 2, graphite heater; 3, BN crucible; 4, sample; 5, thermocouples. (b) Broken as-grown boule: shiny crystal surfaces are visible inside the polycrystalline pellet. (c) Scanning electron micrograph of a $\mathrm{Pt}_{2} \mathrm{HgSe}_{3}$ crystal extracted from the pellet. (d),(e) Top and side views of the crystal structure. In (d) the blue lines indicate the three mirror planes relevant for the topology of the band structure. (f) Sketch of the 3D Brillouin zone. The blue shaded plane is one of the three mirror planes in momentum space. The nodal lines inside the BZ are marked in red. $(\mathrm{g})$ DFT band structure calculation including SOC. (Inset) Enlarged view of the Dirac-like dispersion at $K$, gapped by SOC interactions.

parameters $a=b=7.3482(2)$ and $c=5.2943(1) \AA$, in good agreement with previously reported structural studies $[19,20]$. Measurements of the chemical composition by wavelength-dispersive $\mathrm{x}$-ray spectroscopy in a scanning electron microscope were consistent with the nominal stoichiometry and showed that the $\mathrm{Pt}: \mathrm{Hg}$ ratio is in the window of $1.95 \pm 0.05$ for all samples [20].

Synchrotron-based ARPES measurements were carried out at beam line I05 of Diamond Light Source using photon energies of 19-80 eV [21]. Samples were cleaved in situ along the $a b$ plane at temperatures $<20 \mathrm{~K}$. ARPES data were taken at $\sim 6 \mathrm{~K}$ with energy and momentum resolutions set to $\sim 15 \mathrm{meV}$ and $0.02 \AA^{-1}$, respectively. Densityfunctional theory (DFT) calculations were performed with the QUANTUM ESPRESSO distribution [22,23] using the Perdew-Burke-Ernzerhof functional [24]. Band structures and surface spectral densities were computed by mapping the DFT electronic properties onto maximally localized Wannier functions [25]. For more details, see the Supplemental Material [20], which includes Refs. [26-34].

Bulk jacutingaite consists of layers of $\mathrm{Pt}_{2} \mathrm{HgSe}_{3}$ stacked along the $c$ direction and held together mainly by van der Waals forces [Figs. 1(d) and 1(e)]. Each layer comprises $\mathrm{Pt}$ atoms sandwiched between layers of $\mathrm{Se}$ and $\mathrm{Hg}$ atoms, forming a buckled honeycomb structure. The natural cleavage plane of $\mathrm{Pt}_{2} \mathrm{HgSe}_{3}$ is (001) with a $\mathrm{Hg}$-terminated surface. The structure has an inversion symmetry around the center of the unit cell and three mirror planes perpendicular to the $a b$ plane, related to one another by a $C_{3}$ rotation [blue lines in Fig. 1(d)].

The DFT bulk band structure computed, including spinorbit coupling (SOC), is displayed in Fig. 1(g). In agreement with previous calculations [12,13], we find that $\mathrm{Pt}_{2} \mathrm{HgSe}_{3}$ is a compensated semimetal with a band overlap of $\sim 400 \mathrm{meV}$. At the $K$ and $H$ points, one can readily identify Dirac-like cones, which are gapped by SOC. In bulk $\mathrm{Pt}_{2} \mathrm{HgSe}_{3}$, this gap is only a few $\mathrm{meV}$, in sharp contrast with the large gap found in $\mathrm{ML}_{2} \mathrm{HgSe}_{3}$ at the equivalent point. This suppression is due to a strong interlayer coupling [35], which is also responsible for the large band dispersion found along $k_{z}$. The latter is particularly evident along the $K H$ direction, where the bands forming the gapped Dirac cone cross the Fermi level twice. The nearly parallel dispersion of these two bands along $K H$ further implies that the Dirac cones at $K$ and $H$ are connected by a nodal line, mildly gapped by SOC. Importantly, as emphasized in Ref. [13], DFT calculations show a second nodal line forming a closed loop around the $\Gamma A$ axis inside the BZ, as indicated in Fig. 1(f) by red lines. In the following, we show experimental evidence for the latter and characterize the topological surface state that develops from it.

We start by presenting the Fermi surface of bulk $\mathrm{Pt}_{2} \mathrm{HgSe}_{3}$ as measured with $h \nu=19 \mathrm{eV}$ on the (001) surface [Fig. 2(a)]. These data show well-defined lens-shaped contours on either side of the $M$ points and threefold symmetric features resembling fidget spinners around the $K$ points. Such a coexistence of 1D contours and extended areas of high intensity with sharp boundaries is typical for UV photoemission from highly 3D systems with 2D surface states [36]. It generally arises from surface photoemission interfering with direct transitions that are themselves broadened by the finite $k_{z}$ integration resulting from the strong damping of the final state [36,37]. This can result in an almost complete $k_{z}$ integration of the spectral weight and thus in ARPES data closely resembling a surface projection of the bulk band structure rather than a specific cut through the 3D BZ.

In Fig. 2(b), we summarize the extension of the $k_{z}$ projected Fermi surface pockets determined from 
(a)

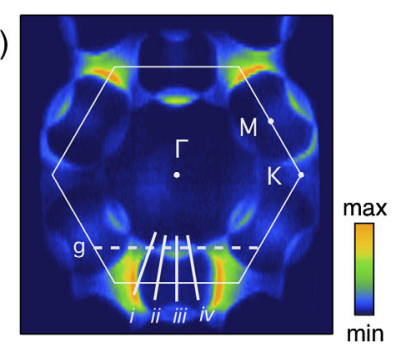

(b)

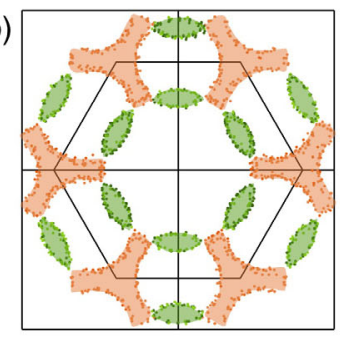

(c)

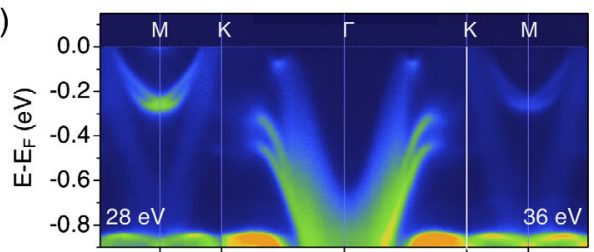

(d)

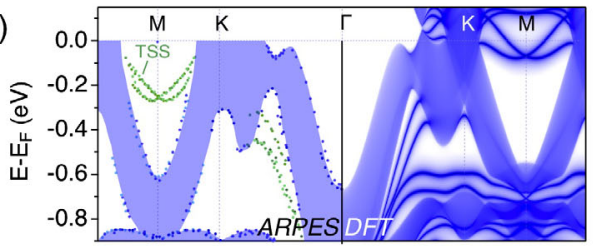

(g)

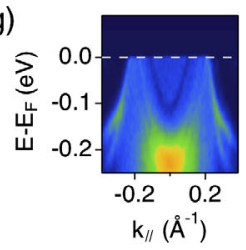

(h) (e)

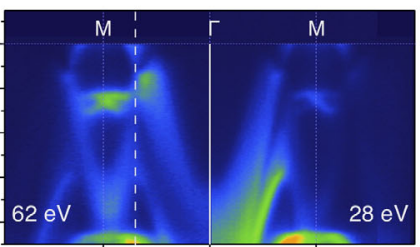

(f)
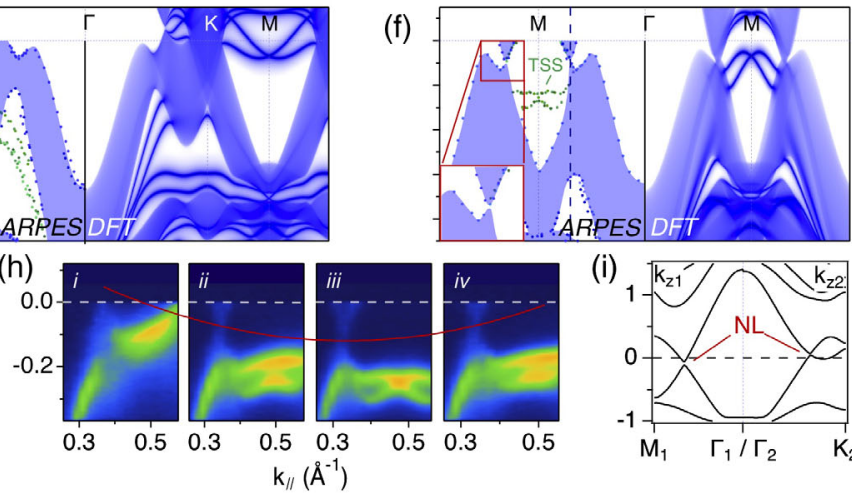

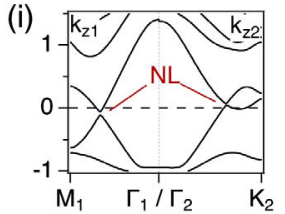

FIG. 2. Bulk and surface electronic structure. (a) ARPES Fermi surface measured with $h \nu=19 \mathrm{eV}$ excitation energy and $p$ polarization. (b) Projected Fermi surface contours of hole (orange) and electron (green) pockets extracted from measurements at 19 and $62 \mathrm{eV}$ photon energies [20]. (c),(e) ARPES dispersion plots. (d),(f) Projected bulk band structure obtained by combining data at 19, 28, 36, and $62 \mathrm{eV}$ photon energies (left half). The dispersion of the surface state is indicated with green dots. DFT calculations of the surface spectral density (right half). (g) Cut along the white dashed line marked on the Fermi surface in (a). (h) Radial cuts across the electronlike lens pocket measured at the positions marked by solid white lines in (a). The dispersion of the nodal line is traced by a red line. (i) DFT bands calculated at $k_{z 1}=-0.2962 \AA^{-1}$ (left) and $k_{z 2}=-0.2735 \AA^{-1}$ (right) showing the nodal line crossings along $\Gamma_{1} M_{1}$ and $\Gamma_{2} K_{2}$.

measurements at different photon energies. Consistent with the semimetallic nature predicted by DFT, we find small pockets of opposite carrier type. The "fidget spinners" at the $K$ points spanning each of them $\sim 6.7 \%$ BZ (orange shaded areas) are holelike, while the lens-shaped pockets with a volume of $\sim 1.5 \% \mathrm{BZ}$ (green shaded areas) that appear to connect the hole pockets are electronlike. We stress that these areas represent the surface projected Fermi surface of a highly 3D system. They are thus reminiscent of extremal orbits, as they are measured in quantum oscillation experiments but cannot be used to deduce the degree of charge compensation. The latter is evident from the DFT dispersion along $K H$, predicting that the fidget spinner hole pocket changes its polarity and becomes electronlike for $k_{z}$ values around $K$ and $H$.

Representative dispersion plots measured along the high symmetry directions $\Gamma K$ and $\Gamma M$ are shown in Figs. 2(c) and 2(e). As on the Fermi surface, the data show a coexistence of sharp 2D bands and broad features, which arise from a surface projection of bands with $k_{z}$ dispersion. Evaluating the edges of the latter in dispersion plots acquired at different photon energies, we obtain the projected bulk band structure displayed as shaded blue areas on the left of Figs. 2(d) and 2(f). These areas are in good agreement with a DFT calculation of the momentumresolved surface density of states along the same path [Figs. 2(d) and 2(f), right]. Both experiment and theory show pronounced minima of the valence band at $\Gamma$ and $M$ and find that the valence band reaches a local maximum just below $E_{F}$ before crossing the Fermi level in the vicinity of $K$. An equally good agreement is observed for several other features that can be identified in experiments. This confirms the interpretation of the ARPES intensity distribution as a bulk projection of the electronic structure and implies a good overall agreement of the full 3D electronic structure of $\mathrm{Pt}_{2} \mathrm{HgSe}_{3}$ with DFT predictions.

We now focus on the nodal line in the bulk electronic structure, which is particularly important for the topological character of $\mathrm{Pt}_{2} \mathrm{HgSe}_{3}$. Moving from $\Gamma$ toward $M$, our data show that conduction and valence bands nearly touch, forming Dirac-like cones at the positions marked by dashed vertical lines in Figs. 2(e) and 2(f) and highlighted in the inset of Fig. 2(f). The radial cuts of this area, displayed in Fig. 2(h), show that the Dirac cone moves in energy but does not noticeably change its small gap. It thus forms a narrowly gapped nodal line that disperses across the Fermi level and forms a loop around $\Gamma A$. In Fig. 2(g), we directly visualize the dispersion of the gapped nodal line by extracting a cut along the long axis of the lenslike electron pocket [dashed line in Fig. 2(a)]. This shows a sharp suppression of intensity in the surface projected spectral weight along a nearly parabolic contour (dark blue in the false color plot) that can be identified with the gap along the nodal line. The width of this suppression suggests a gap of $\sim 25 \mathrm{meV}$ at the bottom of the electron pocket, consistent with the SOC gaps found by DFT.

To illustrate the relevance of this nodal line, we show in Fig. 2(i) band structure calculations along $\Gamma M$ and $\Gamma K$ at 

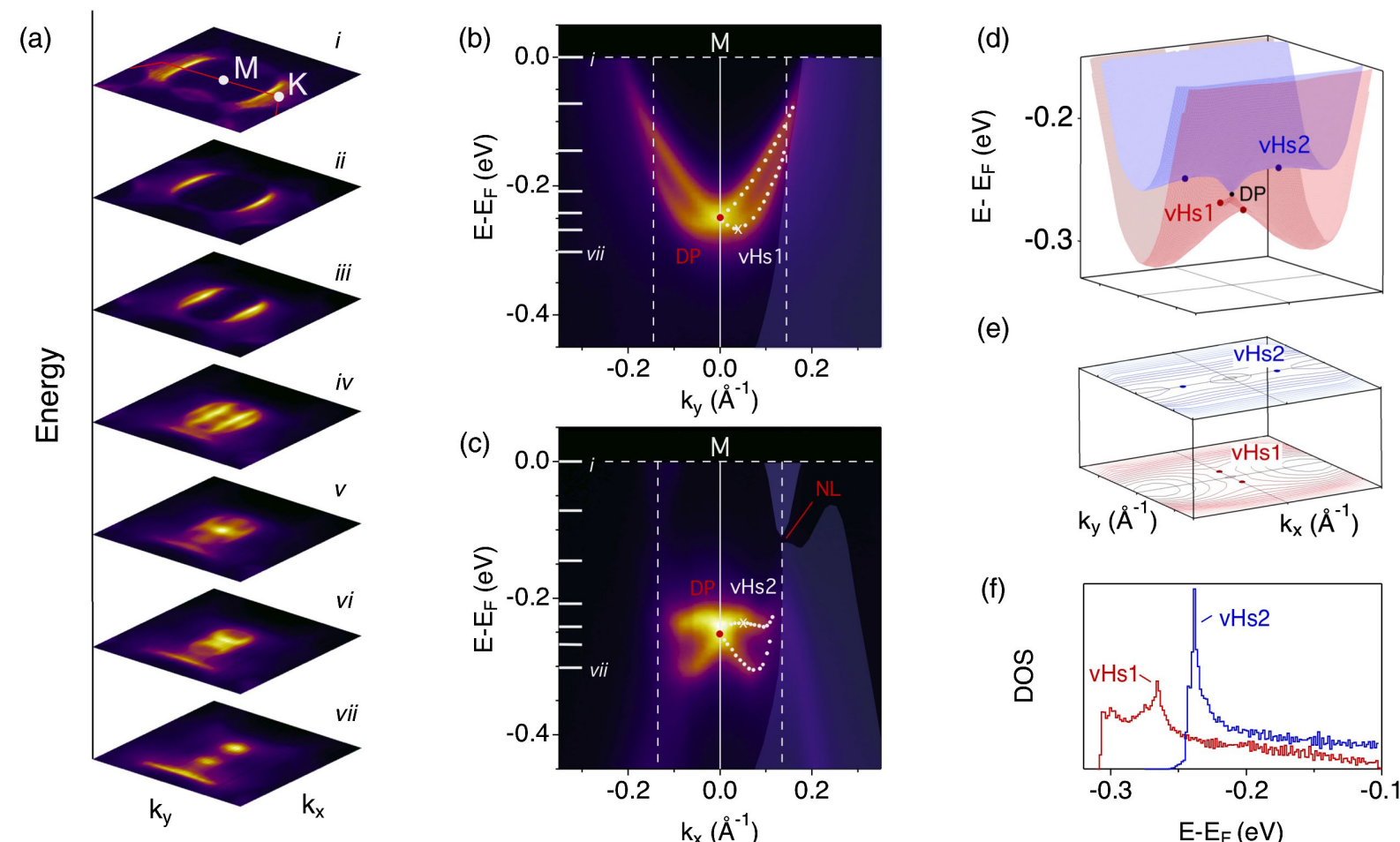

(e)
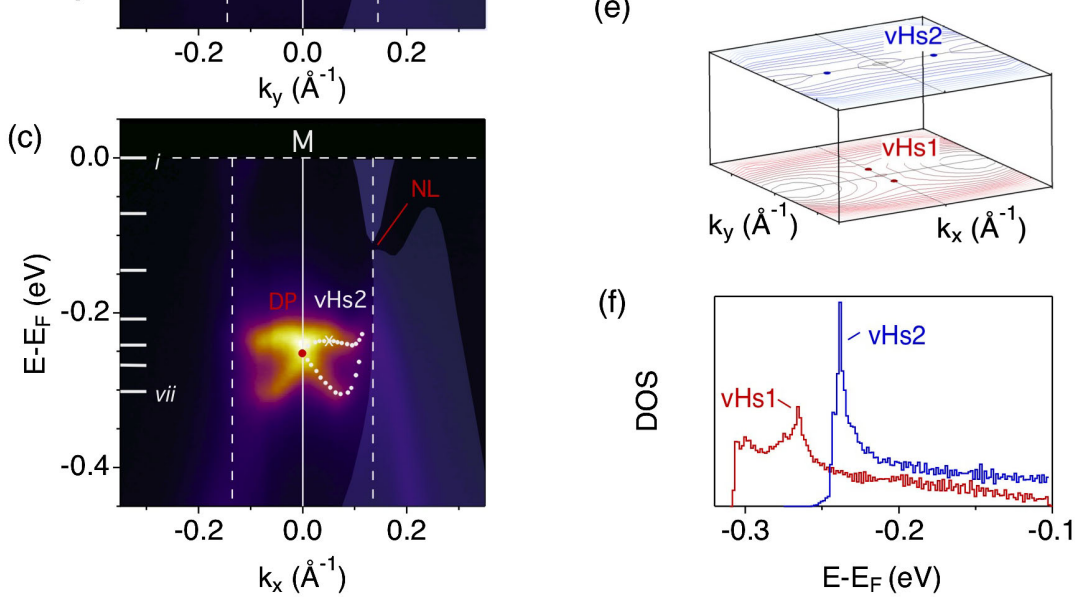

FIG. 3. Dispersion of the topological surface state (TSS). (a) Stack of constant energy maps around the $M$ point. (b),(c) Dispersion along $K M K\left(k_{y}\right)$ and $\Gamma M \Gamma\left(k_{x}\right)$, respectively. On the right-hand side, the extracted surface and bulk band dispersions are superimposed on the data. (d) 3D plot of the energy and momentum dispersion of the TSS extracted over the momentum range indicated by vertical dashed lines in (b) and (c). (e) Associated isoenergy contours of the lower (blue) and upper (red) surface state branches. (f) Histograms of the corresponding $\epsilon(k)$ values, showing sharp peaks at the energy positions of the van Hove singularities.

the $k_{z}$ values with minimal gap. From these calculations, it is evident that the (001) projection of the nodal line separates the surface Brillouin zone in an inner region with trivial band ordering and an outer region comprising the $M$ and $K$ points, which displays a band inversion. Direct calculations show [35] that this band inversion leads to a nontrivial Zak phase [38], thus implying the existence of surface states connecting valence and conduction bands in the outer region of the Brillouin zone.

Inside the projected bulk band gap centered at $M$, we find prominent states with $2 \mathrm{D}$ character that are degenerate at the time reversal invariant $M$ points, but split in pairs away from $M$ before merging into the bulk continuum in the vicinity of the gapped nodal line. These characteristics are hallmarks of surface states. Our calculations, shown on the right-hand side of Figs. 2(d) and 2(f), reproduce the surface states qualitatively and confirm their topological character identified in earlier theoretical work $[12,13,35]$. We note that such calculations do not include surface effects, such as structural relaxations and a possible charge accumulation or depression, and are thus not expected to be in quantitative agreement with experiment. We also find that the exact dispersion of surface states is sensitive to details of the simulations, including the number of bands mapped into Wannier functions [20].
The observation of surface states on the (001) surface of bulk $\mathrm{Pt}_{2} \mathrm{HgSe}_{3}$ is a priori unexpected given that $\mathrm{Pt}_{2} \mathrm{HgSe}_{3}$ can be described as a stack of 2D QSHIs that preserves translational symmetry and thus supports a weak topological phase. Calculations of the $3 \mathrm{D} \mathbb{Z}_{2}$ topological invariants indeed find $(0 ; 001)[12,13,39]$, confirming the weak topological insulator phase generally characterized by gapless modes on the lateral surfaces but fully gapped states on the top and bottom surfaces. Clearly, the surface states found on the (001) surface do not fit this description and thus must be the manifestation of a different topological phase. This phase has been identified in Refs. $[12,13]$ as a TCI, with a nonzero mirror Chern number $\left(\left|C_{m}\right|=2\right)$ associated with the threefold mirror symmetry of the crystal. In such a case, topologically protected surface states are expected on crystal surfaces that preserve the mirror symmetry [40], which is the case for the cleaved (001) surface. The surface state observed by ARPES at the $M$ point can be identified as the signature of such a TCI phase. Taken together with the theoretically robust WTI phase, our results thus provide evidence for the predicted dual topology of $\mathrm{Pt}_{2} \mathrm{HgSe}_{3}$. We note that $\mathrm{Pt}_{2} \mathrm{HgSe}_{3}$ has also been classified among the higher-order topological insulator with the single $\mathbb{Z}_{4}$ indicator equal to $2[39,41]$. Hence, topological surface states are expected not only on 
lateral, top, and bottom surfaces, but might also exist along hinges. However, unlike others systems [42,43], a direct experimental confirmation of hinge states and lateral surface states will be difficult in $\mathrm{Pt}_{2} \mathrm{HgSe}_{3}$, since current approaches only permit the preparation of its (001) surface.

We finally discuss the peculiar dispersion of the TSS. Figure 3(a) shows a stack of constant energy maps of the TSS centered at the $M$ points. Moving from the Fermi level toward higher binding energies, we observe an abrupt change in the distribution of its spectral intensity as the Dirac point is crossed. At low binding energies, the TSS forms pairs of croissant-shaped contours elongated along $k_{x}$ [Fig. 3(a), layers i-iii], whereas at high binding energies the constant energy contours become nearly elliptical with the long axes parallel to $k_{y}$ [Fig. 3(a), layer vii]. A nontrivial dispersion is also evident in the two perpendicular cuts shown in Figs. 3(b) and 3(c). Along $k_{y}$ one recognizes the characteristic dispersion of a Rashba-like spin-split surface state. Along $k_{x}$, however, the upper branch of the TSS has a nonmonotonic dispersion with shallow maxima away from the Dirac point.

To analyze the surface density of states resulting from this unusual dispersion, we first fit the energy position of the two surface state branches in a series of dispersion cuts measured along $k_{y}$ for 20 different values of $k_{x}$, spanning a region of $\pm 0.12 \AA^{-1}$ around $M$. From these fits, we then obtain the hypersurfaces of the energy-momentum dispersion displayed in Fig. 3(d). This analysis demonstrates that, in contrast to the TSSs found in conventional topological insulators $[44,45]$, the TSS on $\mathrm{Pt}_{2} \mathrm{HgSe}_{3}$ has a complex shape with multiple saddle points, resulting in two van Hove singularities in the density of states Fig. [3(e)]. The latter can clearly be identified in the form of sharp peaks at -266 and $-239 \mathrm{meV}$ in the histogram of the $\epsilon(k)$ values for the lower and upper branch, respectively [Fig. 3(f)]. Such singularities were predicted as generic features of TSSs emerging from narrowly gapped line nodes $[13,46]$, but have not been reported before. Tuning these singularities to the Fermi level by bulk or surface doping should favor ferro- or metamagnetic instabilities $[47,48]$ and was recently predicted to stabilize topological superconductivity $[49,50]$.

In conclusion, we reported the synthesis of jacutingaite $\mathrm{Pt}_{2} \mathrm{HgSe}_{3}$ single crystals and provided evidence for their dual-topological nature by comparing the bulk and surface electronic structure determined from ARPES experiments with DFT calculations. The overall agreement with these calculations demonstrates that DFT provides a good description of $\mathrm{Pt}_{2} \mathrm{HgSe}_{3}$. This, in turn, supports the recent DFT prediction of a robust QSHI phase in monolayer $\mathrm{Pt}_{2} \mathrm{HgSe}_{3}$ [10].

We gratefully acknowledge discussions with A. Kuzmenko, A. Morpurgo, C. Renner, and F. Schindler. The experimental work was supported by the Swiss
National Science Foundation (SNSF) (Projects No 200020_165791 and No. 200020_184998). A. M. and N.M. acknowledge support by the NCCR MARVEL of the SNSF. M. G. was supported by the SNSF through the Ambizione program. Simulation time was awarded by CSCS on Piz Daint (production projects s825 and s917) and by PRACE on Marconi at Cineca, Italy (Project ID 2016163963). We acknowledge Diamond Light Source for time on beam line I05 under Proposal No. SI18952. The research data supporting this publication can be accessed at the Yareta repository of the University of Geneva [51].

*anna.tamai@unige.ch

[1] C. L. Kane and E. J. Mele, Phys. Rev. Lett. 95, 226801 (2005).

[2] C. L. Kane and E. J. Mele, Phys. Rev. Lett. 95, 146802 (2005).

[3] B. A. Bernevig, T. L. Hughes, and S.-C. Zhang, Science 314, 1757 (2006).

[4] M. König, S. Wiedmann, C. Brüne, A. Roth, H. Buhmann, L. W. Molenkamp, X.-L. Qi, and S.-C. Zhang, Science 318, 766 (2007).

[5] S. Tang et al., Nat. Phys. 13, 683 (2017).

[6] Z. Fei, T. Palomaki, S. Wu, W. Zhao, X. Cai, B. Sun, P. Nguyen, J. Finney, X. Xu, and D. H. Cobden, Nat. Phys. 13, 677 (2017).

[7] S. Wu, V. Fatemi, Q. D. Gibson, K. Watanabe, T. Taniguchi, R. J. Cava, and P. Jarillo-Herrero, Science 359, 76 (2018).

[8] I. Cucchi, I. Gutiérrez-Lezama, E. Cappelli, S. M. K. Walker, F. Y. Bruno, G. Tenasini, L. Wang, N. Ubrig, C. Barreteau, E. Giannini, M. Gibertini, A. Tamai, A. F. Morpurgo, and F. Baumberger, Nano Lett. 19, 554 (2019).

[9] F. Reis, G. Li, L. Dudy, M. Bauernfeind, S. Glass, W. Hanke, and R. Thomale, Science 357, 287 (2017).

[10] A. Marrazzo, M. Gibertini, D. Campi, N. Mounet, and N. Marzari, Phys. Rev. Lett. 120, 117701 (2018).

[11] M. Z. Hasan and C. L. Kane, Rev. Mod. Phys. 82, 3045 (2010).

[12] J. I. Facio, S. K. Das, Y. Zhang, K. Koepernik, J. van den Brink, and I. C. Fulga, Phys. Rev. Mater. 3, 074202 (2019).

[13] B. Ghosh, S. Mardanya, B. Singh, X. Zhou, B. Wang, T.-R. Chang, C. Su, H. Lin, A. Agarwal, and A. Bansil, Phys. Rev. B 100, 235101 (2019).

[14] M. Eschbach, M. Lanius, C. Niu, E. Młyńczak, P. Gospodarič, J. Kellner, P. Schüffelgen, M. Gehlmann, S. Döring, E. Neumann, M. Luysberg, G. Mussler, L. Plucinski, M. Morgenstern, D. Grützmacher, G. Bihlmayer, S. Blügel, and C. M. Schneider, Nat. Commun. 8, 14976 (2017).

[15] I. P. Rusinov, T. V. Menshchikova, A. Isaeva, S. V. Eremeev, Y. M. Koroteev, M. G. Vergniory, P. M. Echenique, and E. V. Chulkov, Sci. Rep. 6, 20734 (2016).

[16] N. Avraham, A. Norris, Y. Sun, Y. Qi, L. Pan, A. Isaeva, A. Zeugner, C. Felser, B. Yan, and H. Beidenkopf, arXiv:1708 .09062 . 
[17] A. Zeugner, J. Teichert, M. Kaiser, T. V. Menshchikova, I. P. Rusinov, A. V. Markelov, E. V. Chulkov, T. Doert, M. Ruck, and A. Isaeva, Chem. Mater. 30, 5272 (2018).

[18] K. Kandrai, G. Kukucska, P. Vancsó, J. Koltai, G. Baranka, Z. E. Horváth, Á. Hoffmann, A. Vymazalová, L. Tapasztó, and P. Nemes-Incze, arXiv:1903.02458.

[19] A. Vymazalová, F. Laufek, M. Drábek, A. R. Cabral, J. Haloda, T. Sidorinová, B. Lehmann, H. F. Galbiatti, and J. Drahokoupil, Can. Mineral. 50, 431 (2012).

[20] See Supplemental Material at http://link.aps.org/ supplemental/10.1103/PhysRevLett.124.106402 for additional information on sample characterization, ARPES Fermi surfaces at different photon energies and details on the DFT calculations.

[21] M. Hoesch, T. Kim, P. Dudin, H. Wang, S. Scott, P. Harris, S. Patel, M. Matthews, D. Hawkins, S. Alcock, T. Richter, J. Mudd, M. Basham, L. Pratt, P. Leicester, E. Longhi, A. Tamai, and F. Baumberger, Rev. Sci. Instrum. 88, 013106 (2017).

[22] P. Giannozzi et al., J. Phys. Condens. Matter 21, 395502 (2009).

[23] P. Giannozzi et al., J. Phys. Condens. Matter 29, 465901 (2017).

[24] J. P. Perdew, K. Burke, and M. Ernzerhof, Phys. Rev. Lett. 77, 3865 (1996).

[25] N. Marzari, A. A. Mostofi, J. R. Yates, I. Souza, and D. Vanderbilt, Rev. Mod. Phys. 84, 1419 (2012).

[26] K. Lee, É. D. Murray, L. Kong, B. I. Lundqvist, and D. C. Langreth, Phys. Rev. B 82, 081101(R) (2010).

[27] V. R. Cooper, Phys. Rev. B 81, 161104(R) (2010).

[28] G. Prandini, A. Marrazzo, I. E. Castelli, N. Mounet, and N. Marzari, npj Comput. Mater. 4, 72 (2018).

[29] N. Marzari, D. Vanderbilt, A. De Vita, and M. C. Payne, Phys. Rev. Lett. 82, 3296 (1999).

[30] D. R. Hamann, Phys. Rev. B 88, 085117 (2013).

[31] M. J. van Setten, M. Giantomassi, E. Bousquet, M. J. Verstraete, D. R. Hamann, X. Gonze, and G. M. Rignanese, Comput. Phys. Commun. 226, 39 (2018).

[32] A. A. Mostofi, J. R. Yates, G. Pizzi, Y.-S. Lee, I. Souza, D. Vanderbilt, and N. Marzari, Comput. Phys. Commun. 185, 2309 (2014).
[33] Q. Wu, S. Zhang, H.-F. Song, M. Troyer, and A. A. Soluyanov, Comput. Phys. Commun. 224, 405 (2018).

[34] G. Pizzi, A. Cepellotti, R. Sabatini, N. Marzari, and B. Kozinsky, Comput. Mater. Sci. 111, 218 (2016).

[35] A. Marrazzo, N. Marzari, and M. Gibertini, companion paper, Phys. Rev. Research 2, 012063 (2020).

[36] M. Lindroos and A. Bansil, Phys. Rev. Lett. 77, 2985 (1996).

[37] T. Miller, W. E. McMahon, and T.-C. Chiang, Phys. Rev. Lett. 77, 1167 (1996).

[38] J. Zak, Phys. Rev. Lett. 62, 2747 (1989).

[39] M. G. Vergniory, L. Elcoro, C. Felser, N. Regnault, B. A. Bernevig, and Z. Wang, Nature (London) 566, 480 (2019).

[40] T. H. Hsieh, H. Lin, J. Liu, W. Duan, A. Bansil, and L. Fu, Nat. Commun. 3, 982 (2012).

[41] F. Schindler, A. M. Cook, M. G. Vergniory, Z. Wang, S. S. P. Parkin, B. A. Bernevig, and T. Neupert, Sci. Adv. 4, eaat0346 (2018).

[42] F. Schindler, Z. Wang, M. G. Vergniory, A. M. Cook, A. Murani, S. Sengupta, A. Y. Kasumov, R. Deblock, S. Jeon, I. Drozdov, H. Bouchiat, S. Guéron, A. Yazdani, B. A. Bernevig, and T. Neupert, Nat. Phys. 14, 918 (2018).

[43] R. Noguchi et al., Nature (London) 566, 518 (2019).

[44] Y. Xia, D. Qian, D. Hsieh, L. Wray, A. Pal, H. Lin, A. Bansil, D. Grauer, Y. S. Hor, R. J. Cava, and M. Z. Hasan, Nat. Phys. 5, 398 (2009).

[45] Y. Chen, J. G. Analytis, J.-H. Chu, Z. K. Liu, S.-K. Mo, X. Qi, H. J. Zhang, D. Lu, X. Dai, Z. Fang, S. Zhang, I. Fisher, Z. Hussain, and Z.-X. Shen, Science 325, 178 (2009).

[46] B. Singh, X. Zhou, H. Lin, and A. Bansil, Phys. Rev. B 97, 075125 (2018).

[47] J. Liu and L. Balents, Phys. Rev. B 95, 075426 (2017).

[48] B. Binz and M. Sigrist, Europhys. Lett. 65, 816 (2004).

[49] N. F. Q. Yuan, H. Isobe, and L. Fu, arXiv:1901.05432.

[50] X. Wu, M. Fink, W. Hanke, R. Thomale, and D. Di Sante, Phys. Rev. B 100, 041117(R) (2019).

[51] https://yareta.unige.ch/frontend/archive/15906165-9f474410-b1da-aa797d0fe4ef. 